\title{
Impact du colza sur l'environnement : évaluation et limites méthodologiques
}

\author{
Christophe SAUSSE ${ }^{1}$ \\ Nicolas CERRUTTI ${ }^{1}$ \\ Hubert HEBINGER ${ }^{2}$ \\ ${ }^{1}$ CETIOM, \\ Centre de Grignon, \\ Avenue Lucien-Brétignières, \\ 78850 Thiverval-Grignon, \\ France \\ <sausse@cetiom.fr> \\ ${ }^{2}$ CETIOM, \\ Dijon, \\ ZAE Capnord, \\ 10 avenue de Dallas, \\ 21000 Dijon, \\ France
}

Évaluer l'impact environnemental du colza, c'est-à-dire à la fois les apports bénéfiques et les risques générés par la présence sur certains territoires d'une production de colza importante, s'avère d'autant plus difficile que peu d'études ont été réalisées dans ces domaines. En outre, le colza n'occupe qu'une fraction des territoires et se trouve en interaction permanente avec les autres productions agricoles et les milieux naturels. Comment, dans un tel contexte, "isoler » l'effet colza ? Le défi est de taille et I'ensemble des conséquences possibles n'a pas encore été évalué de façon rigoureuse et complète.

Cet article présente l'état des connaissances sur les aspects environnementaux, développés aux échelles de la parcelle et du paysage, et pour les compartiments eau, sol, air et biodiversité. Pour chacun de ces compartiments, les enjeux méthodologiques et limites sont exposés.

\begin{abstract}
The environmental impact of oilseed rape: assessment and methodological limitations
\end{abstract}

This article provides an overview of the impact of oilseed rape on the environment for water, air, soil, and biodiversity. It also questions the concepts and methods used for this evaluation. Oilseed rape has specific characteristics, which may be positive negative or mixed according to the different indicators used for the environmental assessment. But these effects do not allow us to judge the sustainability of the crop itself, because environmental impacts generally result from processes operating at spatial scales (landscape, watershed) and time scales (cropping systems) larger than the field and the crop cycle. This analysis shows that methods designed for the assessment of a single crop or product, like the life cycle analysis, are of poor interest for such topics.

Key words: oilseed rape, environmental impact, water, soil, air, biodiversity

\section{Impacts quantitatifs et qualitatifs sur l'eau}

La consommation en eau du colza ne constitue pas un problème. Les approches type analyse de cycle de vie (ACV) consistant à imputer un contenu d'eau " virtuelle " à une quantité de graines ou d'huile n'ont de sens que dans un contexte où la ressource est limitée et la culture est irriguée (Sausse, 2011). Culture très exceptionnellement irriguée, le colza ne présente pas une menace de ce point de vue. Au contraire, le colza $d$ 'hiver peut se révéler une alternative intéressante dans les territoires où la ressource en eau est limitante au printemps et en été.

\section{Les nitrates}

La question de l'impact de la culture du colza sur la percolation des nitrates a été principalement abordée aux échelles de la parcelle et du système de culture.
Cette question peut être scindée en deux parties.

Le colza est-il un bon précédent? Les reliquats derrière colza sont en général un peu plus élevés que derrière d'autres cultures mais cet inconvénient est compensé par la possibilité de piégeage par les repousses. Le maintien de ces dernières en été permet $d$ 'absorber une bonne partie de l'azote minéral présent dans le sol et de réduire ainsi fortement le risque de lessivage hivernal sous la céréale qui suit. Sous de bonnes conditions, les repousses peuvent piéger jusqu'à 50 unités d'azote en un mois (Reau et Wagner, 2000).

Le colza est-il un bon suivant? Le colza a une réputation justifiée de " pompe à nitrates »: semé tôt, il peut mobiliser des quantités importantes d'azote à l'automne et durant l'hiver, c'est-à-dire pendant la période où le risque de pertes de nitrates par lessivage est le plus sensible. L'azote absorbé par le

Pour citer cet article : Sausse C, Cerrutti N, Hebinger H. Impact du colza sur l'environnement : évaluation et limites méthodologiques. OCL 2012 ; $19(3)$ : 155-163. doi : 10.1684/ocl.2012.0454 
colza à l'automne est à prendre en compte dans le calcul de la fertilisation azotée de printemps.

Peut-on évaluer l'impact au niveau de territoires? Relativement peu de travaux ont été réalisés sur la percolation des nitrates à cette échelle de travail qui implique le recours à des simulations et un important travail d'acquisition voire de modélisation des données d'entrée (pratiques, milieux). Par exemple, le modèle MODCOU utilisé dans le cadre du PIREN Seine (Ledoux et al., 2007) a permis de comparer différentes conditions d'utilisation des cultures intermédiaires pièges à nitrates (CIPAN) et de réduction de la fertilisation azotée. Mais ces scénarios n'intégraient pas de changement $d^{\prime}$ assolement qui aurait permis l'extraction d'un effet " colza ». En revanche, I'étude CLIP (Bonnet et Lorne, 2009) a comparé sur les bassins Seine Normandie et Adour Garonne, différents scénarios d'augmentation des superficies en biocarburants de première et deuxième génération à I'horizon 2030. L'accroissement des flux de nitrate simulés avec l'augmentation des superficies en colza correspond logiquement à une transition depuis des surfaces en prairie et jachère. Mais cette comparaison ne permet pas d'évaluer les conséquences à système de production équivalent, c'est-à-dire ce qui se passerait dans des systèmes de grandes cultures sans conversion de prairie. Ces approches connaîtront probablement un développement important avec l'amélioration des modèles et des possibilités de traitements de l'information géographique.

\section{Les produits phytosanitaires}

L'impact environnemental d'une substance résulte de la combinaison d'une exposition à cette substance et du danger ou effets adverses qu'elle peut provoquer. Le système d'autorisation de mise en marché des produits phytosa- nitaires constitue un filtre qui permet d'éliminer les molécules aux profils écotoxicologiques les plus problématiques. Des indicateurs d'impact sont à l'étude mais restent controversés. Les indicateurs d'exposition, en particulier les taux de pollution et de contamination, sont quant à eux couramment employés. En pratique, les objectifs de gestion sur les bassins versants ne sont pas définis par des indicateurs d'impact, mais par des indicateurs d'exposition comme la pollution, c'est-à-dire le dépassement d'une norme. Différentes normes sont en usage. La concentration de $0,1 \mu \mathrm{g} / \mathrm{L}$ correspond à la norme de potabilité pour une molécule donnée $(0,5 \mu \mathrm{g} / \mathrm{L}$ toutes substances confondues), $2 \mu \mathrm{g} / \mathrm{L}$ à la norme dans l'eau brute destinée à la production d'eau potable $(5 \mu \mathrm{g} / \mathrm{L}$ toutes substances confondues). Enfin, chaque molécule est dotée d'une Norme de Qualité Environnementale (NQE), qui est un seuil de concentration pour juger du bon état des cours d'eau dans le cadre de la Directive Cadre sur l'Eau.

En 2011 on comptait 32 substances actives autorisées à l'usage en culture sur crucifères oléagineuses. Une telle liste est en évolution constante mais elle a connu une décroissance sensible depuis une dizaine d'années à la faveur des renforcements de la réglementation, tant au niveau européen qu'au niveau français (tableau 1). Les données sur la qualité de l'eau fournissent un premier aperçu des pratiques les plus critiques. Le tableau 2 présente des résultats pour la France entière en 2009, pour les molécules utilisées sur colza. Ces chiffres doivent toutefois être interprétés avec prudence. En effet, ils ont une portée indicative, car ils prennent en compte un seuil fixe non réglementaire pour les eaux de surface. De plus, ils incorporent des données issues de réseaux de surveillance générale, visant à donner une représentation fidèle de l'état des masses d'eau, et d'autres issues de

Tableau 1. Décompte de substances actives autorisées à l'usage sur cruciferes oléagineuses (source : index Phytosanitaires ACTA années, 2000, 2005 et 2011).

\begin{tabular}{|llll|}
\hline Types de substance active & $\mathbf{2 0 0 0}$ & $\mathbf{2 0 0 5}$ & $\mathbf{2 0 1 1}$ \\
\hline Insecticides & 29 & 15 & 9 \\
\hline Fongicides & 15 & 10 & 4 \\
\hline Herbicides & 22 & 21 & 18 \\
\hline Divers & & & 1 \\
\hline Total & 66 & 46 & 32 \\
\hline
\end{tabular}

réseaux de surveillance spécifique destinés à suivre des bassins où des problèmes ont déjà été constatés. II existe donc un biais de surestimation. Ces chiffres intègrent par ailleurs l'effet de pollutions accidentelles. L'étude TOPPS menée sur 5 bassins versants a ainsi montré que les pollutions accidentelles pouvaient être à l'origine de $65 \%$ à $95 \%$ de la contamination des eaux ${ }^{1}$. Enfin, beaucoup de molécules utilisées sur colza le sont aussi sur d'autres cultures et ne peuvent de ce fait être complètement attribuées à la culture.

Les pratiques les plus préoccupantes pour la qualité des eaux de surface concernent I'anti-limace métaldéhyde, qui est utilisé sur un grand nombre de cultures, les herbicides de prélevée, et les produits racinaires de post-levée. Herbicides foliaires et insecticides n'apparaissent quasiment pas en raison des propriétés des molécules et/ou de grammages particulièrement faibles. Des fongicides peuvent être retrouvés, ces molécules étant toutefois utilisées sur un grand nombre de cultures. Sur eaux souterraines, les chiffres sont trop faibles pour pouvoir être interprétés et ne tiennent pas compte d'un possible délai entre traitements et contamination des nappes.

Ces constatations générales ne permettent pas d'évaluer les risques au niveau de la parcelle. Une pratique identifiée comme préoccupante au niveau national pourra ne présenter aucune menace localement, car les risques de transfert dans les eaux dépendent des pratiques de traitement (dose, positionnement, équipement, conditions météo), et des caractéristiques propres de chaque parcelle, c'est-à-dire les potentiels d'entrainement vers les eaux superficielles et souterraines. Ces potentiels dépendent d'un grand nombre de variables : façons culturales, type de sol, profondeur, pente, situation par rapport aux cours d'eau, et éventuels aménagements. Pour chaque pratique préoccupante, il s'avère possible de préciser localement les conditions de risque de transfert, ainsi que les solutions. Celles-ci relèvent en générale d'une gestion stratégique (aménagement, programme de traitement), mais la décision tactique de traiter dépend évidemment des conditions météorologiques du moment. II

\footnotetext{
${ }^{1}$ www.topps-life.org
} 
Tableau 2. Présence des molécules utilisées sur colza dans les eaux de surface et souterraines en 2009 sur la France entière (données de base fournies par le ministère de l'Environnement).

\begin{tabular}{|c|c|c|c|c|c|}
\hline \multicolumn{3}{|l|}{ Eaux de surface } & \multicolumn{3}{|l|}{ Eaux souterraines } \\
\hline Molécule & Nombre de stations & $\begin{array}{l}\% \text { de stations } \\
>0,1 \mu \mathrm{g} / \mathrm{L}\end{array}$ & Molécule & Nombre de stations & $\begin{array}{l}\% \text { de stations } \\
>0,1 \mu \mathrm{g} / \mathrm{L}\end{array}$ \\
\hline Métaldéhyde (a) & 827 & $20,56 \%$ & Carbétamide (h) & 3872 & $0,18 \%$ \\
\hline Métazachlore (h) & 1615 & $4,46 \%$ & Métazachlore (h) & 5699 & $0,18 \%$ \\
\hline Tébuconazole (f) & 1500 & $3,53 \%$ & Tébuconazole (f) & 5987 & $0,13 \%$ \\
\hline Propyzamide (h) & 1568 & $3,00 \%$ & Propyzamide (h) & 4440 & $0,09 \%$ \\
\hline Carbétamide (h) & 827 & $2,78 \%$ & Métaldéhyde (a) & 2359 & $0,08 \%$ \\
\hline Azoxystrobine (f) & 1304 & $1,15 \%$ & Napropamide (h) & 4896 & $0,08 \%$ \\
\hline Isoxaben (h) & 993 & $1,11 \%$ & Cyproconazole (f) & 5295 & $0,08 \%$ \\
\hline Cyproconazole (f) & 1477 & $0,88 \%$ & Clomazone (h) & 3358 & $0,06 \%$ \\
\hline Napropamide (h) & 1548 & $0,84 \%$ & Piclorame (h) & 1904 & $0,05 \%$ \\
\hline Clomazone (h) & 1549 & $0,71 \%$ & Metconazole (f) & 2325 & $0,04 \%$ \\
\hline Metconazole (f) & 571 & $0,35 \%$ & & & \\
\hline Cyperméthrine (i) & 968 & $0,31 \%$ & & & \\
\hline
\end{tabular}

Ce tableau est indicatif et ne permet pas de représenter l'état des masses d'eau en raison d'importants biais d'échantillonnage, et du choix du seuil arbitraire de $0,1 \mu \mathrm{g} / \mathrm{L}$ qui est en général inférieur aux normes de qualité environnementales utilisées pour les eaux de surface dans le cadre de la Directive-cadre sur l'Eau. Les molécules listées sont celles autorisées sur colza en 2009, pour lesquelles existe au moins une station où ce seuil est dépassé au moins une fois dans l'année. Entre parenthèses, le type de traitement (a : anti-limace ; $f$ : fongicide ; h : herbicide ; i : insecticide).

est déconseillé de traiter par fort vent, ou si une forte pluie est attendue (d'autant plus sur les parcelles à fort risque de ruissellement).

Les possibilités de transfert de produits phytosanitaires et les solutions qui peuvent y être apportées doivent se raisonner à l'échelle du système de culture et du territoire. L'adaptation des pratiques de désherbage se raisonne à la rotation. Une réduction de l'usage des herbicides nécessite une approche de reconception des systèmes de culture, avec l'allongement des rotations et des techniques de gestion du stock semencier (faux semis, gestion des intercultures). De la même façon, substituer des produits en fonction de leur potentiel de contamination des eaux ne peut pas se décider sans réfléchir à la cohérence sur la rotation. Par exemple, dans le cas des herbicides racinaires de post-levée, leur substitution par des herbicides foliaires peut aggraver la probabilité d'apparition de graminées résistantes. Au niveau du territoire, les aménagements du parcellaire et l'organisation spatiale des cultures jouent un rôle dans la lutte contre les ruissellements. Les zones tampons permettent l'infiltration de l'eau. La coordination des assolements pour éviter des blocs de culture homogène est également un moyen de lutte efficace.

\section{Impacts sur les sols}

\section{Érosion}

Culture d'hiver présentant le cycle le plus long parmi les cultures annuelles, le colza présente l'avantage d'offrir une longue couverture du sol ce qui est un atout pour lutter contre l'érosion. Le taux de couverture est toutefois variable à l'automne selon les régions et scénarios climatiques.

En complément, des aménagements anti-érosifs et l'adoption de pratiques d'implantation innovantes, basées sur une moindre surface de sol travaillée et/ ou des cultures associées, permettraient de renforcer encore les prédispositions naturelles de la culture à protéger les sols.

La carte d'aléas d'érosion des sols en France (figure 1) permet de bien identifier les zones de grandes cultures exposées où le colza peut jouer un rôle positif. II s'agit des régions au nord de Paris où la culture a fortement progressé depuis 10 ans, et le Sud-Ouest où la culture connaît une marge de progression.

\section{Compaction}

Le phénomène de compaction des sols est lié à la teneur en eau du sol lors des interventions culturales, au poids des machines, au type de pneus et à leur pression de gonflage, aux outils mis en œuvre, ainsi qu'à la fréquence des passages. Culture implantée et récoltée en été, le colza n'est pas, parmi les grandes cultures, celle qui expose les sols aux risques de compaction les plus grands. Seule la fréquence des passages un peu supérieure constitue un facteur aggravant.

C'est incontestablement lors des apports d'azote en fin d'hiver que les risques sont les plus élevés, ainsi que lors des récoltes si celles-ci se déroulent en conditions de sol humide. Mais dans ce dernier cas, les risques sont au plus comparables à ceux des céréales.

\section{Qualité des sols}

La notion de " qualité du sol " renvoie à ses fonctions (Doran et Parkin, 1994), c'est-à-dire la capacité du sol à 1) soutenir la productivité biologique, 2) garantir la sécurité sanitaire des plantes, animaux, et consommateurs, 3) atténuer des dommages à l'environnement (par exemple fonction de dégradation des produits phytosanitaires). Cette notion englobe et dépasse le concept de fertilité, traditionnellement associé à la productivité des cultures. Mais elle ne rend pas forcément compte 


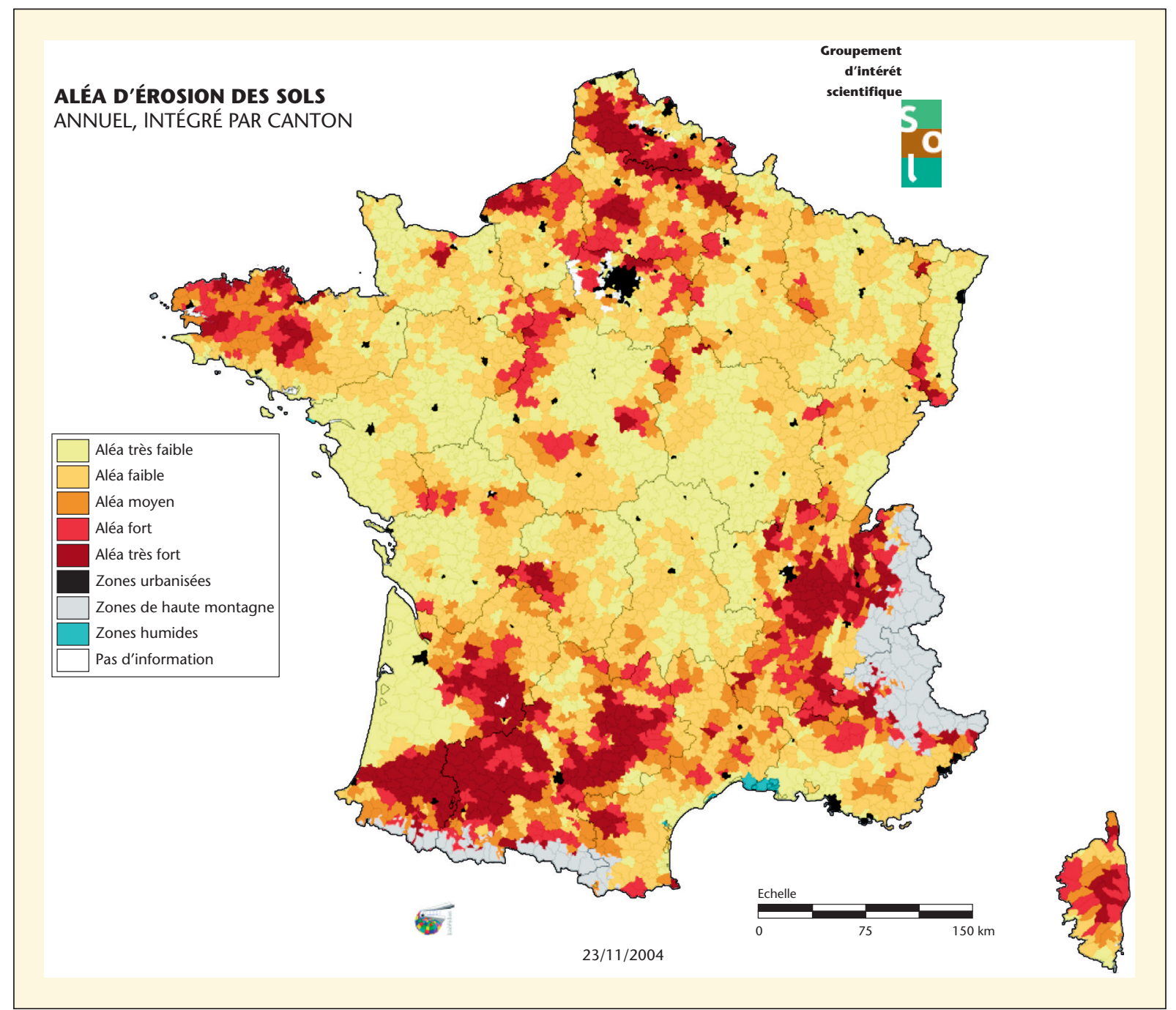

Figure 1. Carte d'aléa d'érosion des sols en France à l'échelle des cantons (source Gis Sol, INRA, 2004).

de la relativité du jugement qu'il convient de porter sur les sols. En effet, I' " aptitude culturale " d'un sol, terme plus précis que celui de "fertilité ", n'a de sens que pour un système de culture donné (Sébillotte, 1989). Pour illustrer cette idée, nous pouvons citer la Champagne anciennement "Pouilleuse " qui est devenu une importante région de grande culture après I'apparition des engrais chimiques. Cette analyse montre le danger qu'il y a à qualifier la qualité d'un sol dans I'absolu, indépendamment d'un diagnostic local, prenant en compte le contexte pédoclimatique et le système de culture. La vraie question est plutôt de savoir si le sol offre les services attendus dans un contexte donné. Ces services dépendront de diverses caractéristiques physico-chimiques et biologiques des sols. Les premières sont familières en agronomie et les secondes moins bien connues. La qualité des sols dépend donc d'un grand nombre de facteurs qui ne sont pas intrinsèquement liés à une culture donnée. Toutefois, le colza présente des caractéristiques physiologiques qui peuvent avoir un impact sur le sol et les fonctions qui lui sont associées. Le colza est une plante riche en glucosinolates, qui grâce à l'enzyme myrosinase peuvent être dégradés en substances affectant la microflore du sol. Le projet MICROGER (Martin et Lemanceau, 2008) a montré que la culture du colza peut ainsi entraîner des modifications importantes des communautés microbiennes, ainsi que de la qualité phytosanitaire du sol en diminuant le potentiel infectieux de Rhizoctonia solani. D'autres effets allélopathiques ont été mis en évidence en conditions contrôlées sur Aphanomyces du pois et le piétin échaudage du blé (Reau et al., 2005), mais les effets au champ restent toutefois difficiles à quantifier.

Le colza absorbe de manière efficace les métaux lourds, y compris certains isotopes radioactifs, ce qui est mis à profit sur un site pilote à Tchernobyl pour rendre à nouveau le sol cultivable (Kostioukevitch, 2010) ! Ce potentiel de phytoremédiation des sols pollués peut être utilisé dans des situations bien particulières, avec toutes les précautions $\mathrm{d}^{\prime}$ usage que cela requiert.

\section{Impacts sur I'air}

L'impact du colza sur la qualité de l'air concerne principalement les produits 
phytosanitaires. Les risques allergiques liés au pollen sont parfois évoqués, mais semblent négligeables. La floraison du colza intervient au même moment que celles d'autres espèces allergisantes (bouleau, dactyle...), et son odeur particulière peut faire croire aux personnes allergiques à une relation de cause à effet fortuite. Faveret (1976), a constaté une sensibilisation infra-clinique au pollen de colza chez $50 \%$ de sujets cliniquement atteints de pollinose de printemps. Mais cette sensibilisation aboutit à une pollinose dans $2 \%$ des cas. II conclut au caractère exceptionnel des allergies au pollen de colza. Aucune donnée récente n'est venue remettre en cause ce constat. Aujourd'hui, le pollen de colza ne fait plus partie de la batterie de test standard chez les allergologues.

L'exposition aux produits phytosanitaires dans l'air fait l'objet de suivi dans plusieurs régions. La trifluraline, désherbant largement utilisé sur colza, y était retrouvée fréquemment avant l'interdiction de son utilisation en 2008. Une étude d'ATMO en Région Poitou Charente a fait le point en 2009 dans des zones rurales et urbaines contrastées vis-à-vis de la pression en colza dans le paysage (ATMO Poitou-Charentes, 2009). La zone rurale est globalement davantage exposée aux produits phytosanitaires, ce qui traduit un effet de proximité des cultures. Sont principalement retrouvés des herbicides, y compris le métazachlore utilisé en prélevée du colza. Mais les pics de pollution ont lieu au printemps, en raison principalement de la pendiméthaline utilisée sur maïs et tournesol. Les expositions sont moindres pour les fongicides et faibles pour les insecticides, ces derniers étant analysés à des doses inférieures à celles rencontrées en ville.

Les principales mesures à prendre pour éviter la dispersion des produits dans I'air consistent à utiliser un matériel adapté (buses antidérives) et à réaliser les traitements dans de bonnes conditions météorologiques. Le vent accroît la dérive des produits, mais aussi leur volatilisation.

\section{Impact sur la biodiversité}

\section{Élements généraux}

La biodiversité représente, tout d'abord, une valeur en tant que telle et participe de notre cadre de vie. Elle est aussi à l'origine de services écosystémiques, comme la régulation des ravageurs (biodiversité fonctionnelle).

L'impact du colza sur la biodiversité est une question complexe. Un premier niveau d'analyse consiste à partir de la parcelle. De ce point de vue, le jugement peut porter sur le colza comme habitat plus ou moins favorable, en distinguant ce qui relève des caractéristiques de la plante et de l'impact des pratiques. Un second niveau d'analyse concerne le paysage dans lequel le colza s'insère. La biodiversité résulte en effet de l'interaction entre différents habitats proches, par exemple la parcelle et une bande enherbée, ou lointains (figure 2). L'étude de la biodiversité doit également prendre en compte la dimension temporelle. Même pour des espèces à cycle court et inféodées à la parcelle, comme certaines espèces de carabes, l'échelle de temps pertinente pour étudier l'impact des pratiques est celle du système de culture : pour interpréter les observations sur une culture donnée, il convient de connaître I'historique cultural de la parcelle, en particulier précédents et travail du sol.

Le colza est un habitat au sens écologique du terme : il peut répondre aux besoins de certaines espèces pour le gîte et le couvert, mais se révèle moins favorable pour d'autres dont les exigences sont différentes. Feuillage, fleurs

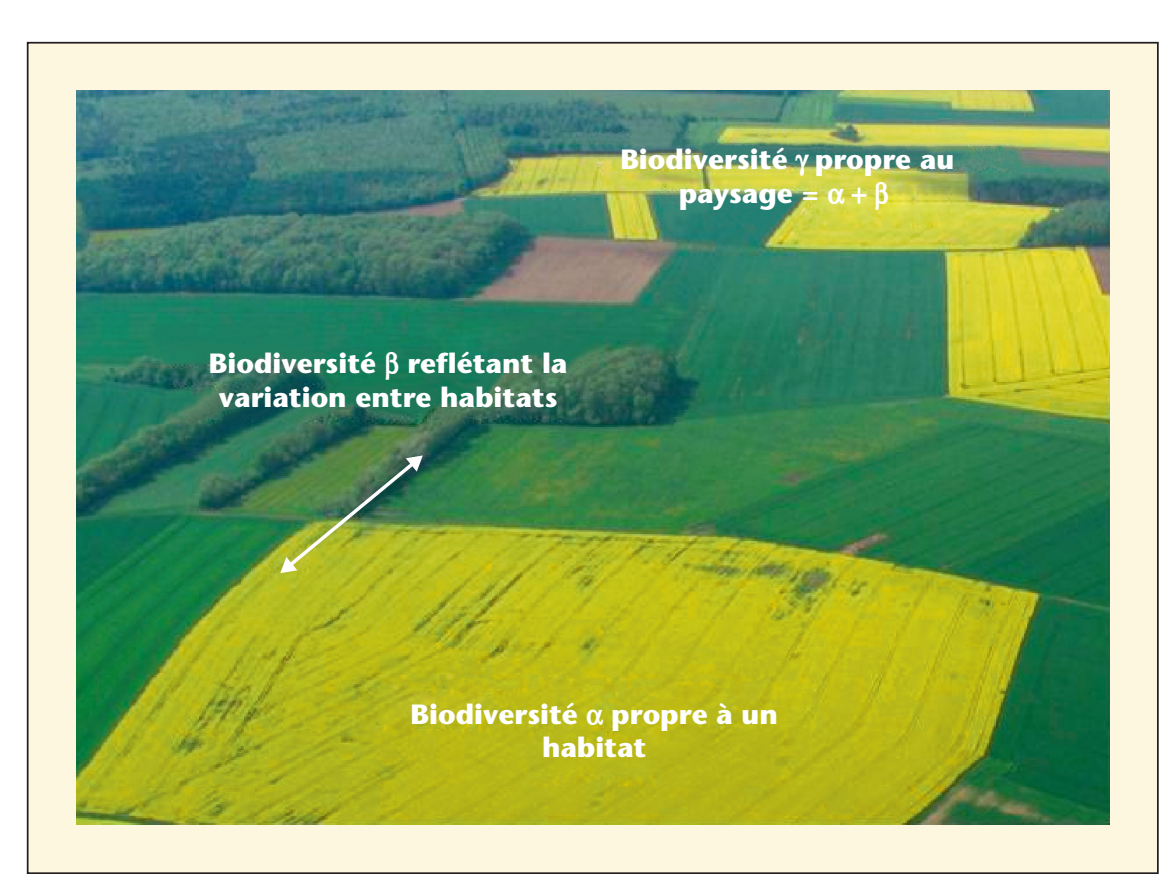

Figure 2. Décomposition schématique de la biodiversité. et graines du colza constituent des ressources trophiques. Le colza est particulièrement attirant pour de nombreuses espèces d'insectes nuisibles ou utiles, voire élevées par I'homme (cas des abeilles domestiques). Adultes et larves de ces insectes peuvent constituer des proies pour d'autres espèces comme des oiseaux. Le colza offre une couverture du sol particulièrement longue, appréciée par certaines espèces d'insectes, par exemple les carabes dont certains sont auxiliaires des cultures. Le gibier y trouve un refuge quand le couvert est suffisamment développé. Les grands mammifères peuvent $s^{\prime} y$ cacher en fin de cycle et causer des dégâts tout particulièrement en hiver (piétinement, pâturage des cervidés). La couverture hivernale du sol est en revanche défavorable pour certaines espèces qui se nourrissent en automnehiver sur chaumes non travaillés, comme certains oiseaux, par exemple l'alouette des champs. Mais ce problème n'est pas spécifique au colza et doit être abordé à l'échelle du paysage, car il concerne l'équilibre entre cultures d'hiver et de printemps dans la sole.

Le colza est parfois incriminé pour ses nombreux traitements insecticides qui induiraient un accroissement de la mortalité directe et indirecte de certaines espèces. Ce constat repose sur un raisonnement global impliquant la toxi- 
cité aiguë ou chronique des produits sur des organismes non cibles, ainsi que les effets induits sur la chaîne trophique (réduction des ressources consommables par des taxons situés plus haut dans la chaîne alimentaire). L'expertise scientifique collective "Agriculture et biodiversité " (Burel et Garnier, 2008) a toutefois montré la difficulté de tirer des conclusions sur ce sujet, en considérant la biodiversité dans son ensemble aux échelles du système de culture et du paysage. Les études sont en effet fragmentaires et concernent principalement des couples molécules-taxons. Dresser un tableau global pose des problèmes de définition des systèmes étudiés. Comment représenter la biodiversité ? Quelle échelle spatiotemporelle considérer? Des approches holistiques sont cependant possibles. Par exemple, Hole et al. (2005) ont revu les études visant à comparer exploitations bio et conventionnelles. Mais les conclusions restent globales, sans que I'on puisse distinguer les effets de tel ou tel facteur. Les résultats sont contrastés suivant les études et les taxons, avec des effets positifs dans deux tiers des cas. Mais les auteurs soulignent qu'il est difficile d'interpréter les résultats: les différences de biodiversité résultentelles de différences de conduites de culture, de paysage (les exploitations bio ont tendanciellement de petites parcelles), ou de l'imbrication des deux types d'exploitation (" surface critique " de bio dans le paysage pour observer des effets). Les problèmes méthodologiques sont encore plus aigus pour identifier l'impact des pratiques culturales dans un système de production donné. Nous sommes ici sur un front de recherche mouvant et très actif, mais qui n'a pas encore permis d'obtenir des résultats probants. En l'état actuel des connaissances, il est difficile de se prononcer sur l'impact global des traitements insecticides réalisés sur colza. Dans le doute, l'attitude raisonnable consiste à éviter tout traitement inutile d'autant plus que le rôle régulateur de la faune auxiliaire (biodiversité fonctionnelle) n'est plus à prouver. À l'avenir, la multiplication des réseaux de surveillance et de suivi post-homologation permettra d'acquérir de nouvelles données précises pour améliorer notre connaissance des impacts.

Le colza est implanté en non-labour dans $52 \%$ des cas (enquête du CETIOM sur les pratiques culturales 2010, non publiée). Cette pratique est réputée favorable à la biodiversité du sol. Cela a été vérifié pour les lombrics (Pelosi, 2008). Mais le non-labour englobe en réalité une grande variété de pratiques plus ou moins perturbatrices de la structure du sol.

Ces considérations sur les propriétés plus ou moins favorables du colza doivent être replacées dans un contexte plus global. En effet, la biodiversité résulte principalement de la diversité et de l'agencement des habitats au sein des paysages: la question est de savoir comment le colza $s^{\prime} y$ insère, avec les autres grandes cultures, et d'autres habitats naturels ou créés par I'homme. L'impact de la configuration du paysage sur la biodiversité fait l'objet d'une abondante littérature scientifique et technique. Des approches pragmatiques permettent aujourd'hui $\mathrm{d}^{\prime}$ aménager le parcellaire en fonction d'objectifs locaux, visant à privilégier certaines espèces emblématiques ou plus prosaïquement la biodiversité ordinaire, avec le pari qu'elle favorise les auxiliaires de culture. Les leviers sont le choix d'espèces adaptées pour les haies et bandes enherbées, le dimensionnement, la localisation et le mode de gestion de ces infrastructures, la taille des parcelles ainsi que l'organisation spatio-temporelle des cultures. L'idée force est d'introduire une diversité des éléments paysagers.

\section{Impacts du colza sur la biodiversité aviaire}

Le colza est un couvert réputé favorable pour certaines espèces $d^{\prime}$ oiseaux comme le bruant des roseaux (Burton et al., 1999) et la linotte mélodieuse (Moorcroft et al., 2006). Mais quelques espèces ne sauraient résumer la biodiversité. Evaluer l'impact de la culture de colza requiert des indices plus intégrateurs comme l'abondance en oiseaux spécialistes des espaces agricoles, le Farmland Bird Index, qui est l'un des indices de la biodiversité les plus étudiés, et a été retenu comme indice structurel du développement durable par I'Union Européenne. Au sommet de la pyramide alimentaire, les oiseaux peuvent être affectés par des perturbations occasionnées aux niveaux inférieurs. Aussi, leur état reflète-t-il bien celui de l'ensemble des espèces (insectes, végétaux) dont ils dépendent.
En vue de réaliser un diagnostic de l'impact des cultures d'oléagineux sur les populations d'oiseaux, le CETIOM a initié une étude en collaboration avec I'INRA et le Muséum National d'Histoire Naturelle (MNHN). Des bases de données nationales ont servi à analyser les relations entre I'utilisation du territoire des petites régions agricoles et quatre indices de biodiversité aviaires : l'abondance des spécialistes agricoles, la nombre d'espèces, un indice de spécialisation des communautés indiquant si les oiseaux observés sont inféodés à certains type de milieu ou généralistes, et un indice trophique reflétant la diversité des régimes alimentaires (Sausse et al., 2011). Ces indices ont été fournis par le programme STOC (Suivi temporel des oiseaux communs coordonné par le MNHN). L'étude menée sur des données 2004 a couvert 182 petites régions agricoles où le colza est cultivé, chacune d'entre elles étant décrite par les variables suivantes: pourcentage de colza, de céréales, de prairies et d'espaces semi-naturels dans le territoire.

Les résultats montrent que les pourcentages de colza sont positivement corrélés à l'abondance et à l'indice de spécialisation, mais négativement à l'indice trophique. Aucun lien n'est constaté avec la richesse spécifique. Toutefois la variabilité des indices de biodiversité aviaire n'est expliquée que partiellement par les variables retenues (aux alentours de $15 \%$ ). La pression en colza n'est donc pas un facteur explicatif majeur. La présence importante de colza dans une région $n^{\prime}$ implique pas de baisse de la biodiversité aviaire. Cette étude exploratoire montre que le diagnostic doit prendre en compte d'autres facteurs que la simple occupation du territoire, par exemple l'impact des pratiques culturales et des aménagements agroécologiques.

\section{Colza et pollinisateurs}

Le colza est une plante mellifère et pollinifère, très attractive pour les pollinisateurs (Holzshuh et al., 2011) et par conséquent très visitée (Mesquida et al., 1988). Les relevés de terrain indiquent que I'abeille domestique Apis mellifera $\mathrm{L}$. représente environ $85 \%$ des individus collectés sur les parcelles, les autres pollinisateurs présents étant par ordre d'importance les bourdons, les diptères 
et les abeilles solitaires. Cette culture, de par sa floraison abondante et continue, apporte du nectar et du pollen de qualité et favorise ainsi la reprise d'activité printanière des abeilles. Elle représente donc une culture incontournable pour l'abeille domestique et par conséquent pour l'apiculture. C'est environ $35 \%$ de la production de miel française qui est élaborée à partir des cultures oléagineuses, notamment le colza et le tournesol. A titre d'exemple, une ruche à proximité d'une parcelle de colza peut produire jusqu'à $40 \mathrm{~kg}$ de miel si les conditions climatiques sont favorables au butinage.

$C^{\prime}$ est parce qu'elle génère une activité économique grâce à l'élaboration des divers produits de la ruche (miel, pollen, gelée royale, propolis, cire) que l'abeille domestique est la mieux connue des 20000 espèces d'abeilles présentes dans le monde. C'est également une espèce emblématique dont I'homme récoltait déjà le miel il y a plus de 10000 ans. Ce rapport de proximité a attiré l'attention sur le déclin du cheptel apiaire. Par extension, les chercheurs se sont aperçu que l'ensemble des pollinisateurs était en danger à l'échelle mondiale (Gallai et al., 1999). Les travaux actuels mettent en avant plusieurs causes possibles de ce déclin dont les principales seraient : les parasites et maladies des abeilles, la raréfaction et I'appauvrissement qualitatif des ressources alimentaires liés à l'uniformisation des paysages agricoles et à la diminution de la biodiversité floristique, l'exposition aux produits phytosanitaires. Certains de ces facteurs pourraient, selon des études récentes, entrer en synergie (Vidau et al., 2011).

Mais l'évaluation de l'état de santé des pollinisateurs en environnement de grande culture de colza est une problématique complexe dans la mesure où les aires de butinages sont souvent vastes (plusieurs kilomètres carrés pour les espèces les plus grosses) et incluent par conséquent d'autres cultures ainsi que des éléments fixes du paysages comme les haies, les bosquets qui ont parfois une valeur attractive pour ces insectes supérieure à celle du colza. Aussi, les abeilles sont des organismes très sensibles à la qualité de leur environnement qui se retrouvent en contact avec de nombreux polluants présents dans l'air, dans l'eau ou sur les végétaux. Ceux-ci résultent des activités humaines ayant lieu sur un territoire et notamment des pratiques agricoles effectuées sur diverses cultures. L'abeille domestique est souvent prise comme indicateur car on connait sa biologie et on sait l'élever. De plus, la ruche est une unité d'étude ayant sa dynamique propre (que l'on peut mesurer) et dans laquelle on peut réaliser des prélèvements.

La méthodologie employée pour connaitre la dynamique de colonies d'abeilles domestiques à proximité de champs de colza se décline en deux actions complémentaires : la collecte de variables à l'intérieur des colonies (pression en pathogènes, résidus de produits phytosanitaires en lien avec les niveaux de population et les espèces butinées) et une évaluation de la fréquentation des cultures via des comptages à l'intérieur des parcelles lors du butinage. Depuis 2010, le CETIOM est impliqué dans ce type de suivi et poursuit aujourd'hui ce travail en partenariat avec I'ITSAP-Institut de l'Abeille pour structurer un observatoire pérenne de ruchers en environnement de grandes cultures oléagineuses (colza et tournesol). À ce jour, des données sont collectées régulièrement sur le terrain et intègrent des variables très diverses afin de prendre en compte les nombreux facteurs qui peuvent influer sur l'état de santé des colonies (pratiques agricoles, apicoles, environnement des ruchers, maladies...). Les résultats sont cependant difficiles à interpréter en raison des nombreux facteurs entrant en ligne de compte et des environnements variables.

Afin de limiter les effets non intentionnels sur les abeilles des traitements phytosanitaires, une réglementation stricte a été mise en place et encadre leur usage sur le colza. Dès l'apparition des premières fleurs sur la culture, seuls les produits dont l'usage est autorisé pendant la floraison peuvent être appliqués et uniquement en l'absence d'abeilles. Les producteurs sont tenus de respecter les seuils de nuisibilité ainsi que les conditions d'emploi associées à l'usage des produits telles que mentionnées sur l'étiquette et de proscrire les mélanges pyréthrinoides/triazoles ou imidazoles qui sont strictement interdits. L'exposition aux insecticides ayant une toxicité aiguë importante vis-à-vis des abeilles est réduite par le respect de la règlementation et des bonnes pratiques agricoles. Toutefois, le danger qui pourrait résulter de l'exposition des abeilles à des faibles doses de produits phytosanitaires dans I'environnement et les interactions possibles avec d'autres facteurs de stress tels qu'évoqués précédemment est une problématique complexe à étudier du point de vue scientifique, et pour laquelle les chercheurs se heurtent à des questions d'ordre méthodologique. Ces considérations dépassent largement le cadre de la culture du colza et démontrent l'intérêt d'une démarche inter-filières et pluridisciplinaire pour avancer vers la compréhension des facteurs de pression qui s'exercent sur les pollinisateurs.

Si la culture du colza représente, nous l'avons vu, une ressource pour les pollinisateurs, il faut également considérer que ces derniers sont favorables à la production dans la mesure où ils apportent un service écosystémique. Cela amène à la prise de conscience de l'intérêt pour les filières de travailler conjointement pour une meilleure prise en compte des pollinisateurs dans les itinéraires techniques. En culture de colza semences, $95 \%$ de la production grainière est assurée par les insectes pollinisateurs (Pierre et Renard, 2010). L'alternance de bandes mâles fertiles et mâles stériles rend cette production dépendante des insectes vecteurs de pollen car une fécondation croisée est nécessaire. On sait en effet que $75 \%$ du pollen total est capturé à moins de six mètres du point d'émission. Ainsi, à plus de 24 mètres la pollinisation anémophile n'assure que 1 à $4 \%$ de la production grainière (Mesquida et Renard, 1981). Pour garantir un rendement en graines et aussi des aspects qualitatifs comme le calibre par exemple, la production de semences nécessite un renforcement en pollinisateurs par disposition de ruches à proximité des parcelles.

Dans le cas du colza mâle fertile, destiné à la consommation, la dépendance visà-vis des pollinisateurs est moindre. En effet, la fécondation anémophile est majoritaire et les plantes compensent les déficits en pollinisateurs en augmentant le nombre de fleurs ainsi que la longueur des ramifications secondaires et tertiaires. Cependant, il est important de noter que le pollen de colza est non pulvérulent et par conséquent peu adapté au transport aérien. Pierre et al. (2010) et Pierre et Renard (2010) montrent que les insectes permettent la mise en suspension du pollen dans l'air ce qui 
les amène à proposer le concept d'une pollinisation anémophile assistée par les insectes. Ce n'est donc pas seulement en déposant du pollen sur les stigmates que les insectes contribuent à la fécondation des fleurs mais aussi de manière indirecte en favorisant la dispersion du pollen dans I'air. Pierre et Renard (2010) indiquent qu'ainsi, les insectes contribuent à la production de graines à hauteur de $36 \%$ en colza de consommation. De plus, les expérimentations conduites sur du colza d'hiver et de printemps montrent une modification de l'architecture des plantes en présence de pollinisateurs (Mesquida et Renard, 1981 ; Lerin et Rivault, 1982 ; Mesquida et al., 1988 ; Sabbahi et al., 2005). Plusieurs impacts du butinage ont été répertoriés :

- diminution des ramifications secondaires et tertiaires ;

- diminution du nombre de fleurs et de leur durée de vie ;

- augmentation du nombre de siliques par plante ;

- augmentation du nombre de graines par siliques ;

- diminution du poids de mille grains ;

- maturation plus homogène des siliques.

Néanmoins, selon la méthodologie employée dans les expériences, les gains de rendement divergent et sont beaucoup plus flagrants sur le colza de printemps en raison de son cycle court et de ses capacités de compensation faibles.

\section{Conclusion}

L'évaluation de l'impact du colza sur l'environnement se heurte à deux difficultés méthodologiques.

Les indicateurs offrent des simplifications, obligatoires pour des raisons pratiques, mais parfois peu pertinentes. Par exemple, il peut y avoir confusion entre usage, exposition et impact. Les simplifications concernent également les objets évalués. Ainsi, la sensibilité à I'entraînement vers les eaux des produits phytosanitaires varie beaucoup, ce qui rend risqué l'usage de valeurs moyennes localement inadaptées. Ce problème de simplification est encore plus aigu dans le cas de la biodiversité, car il faut choisir des espèces particulières pour la représenter, ce qui peut entraîner des biais.

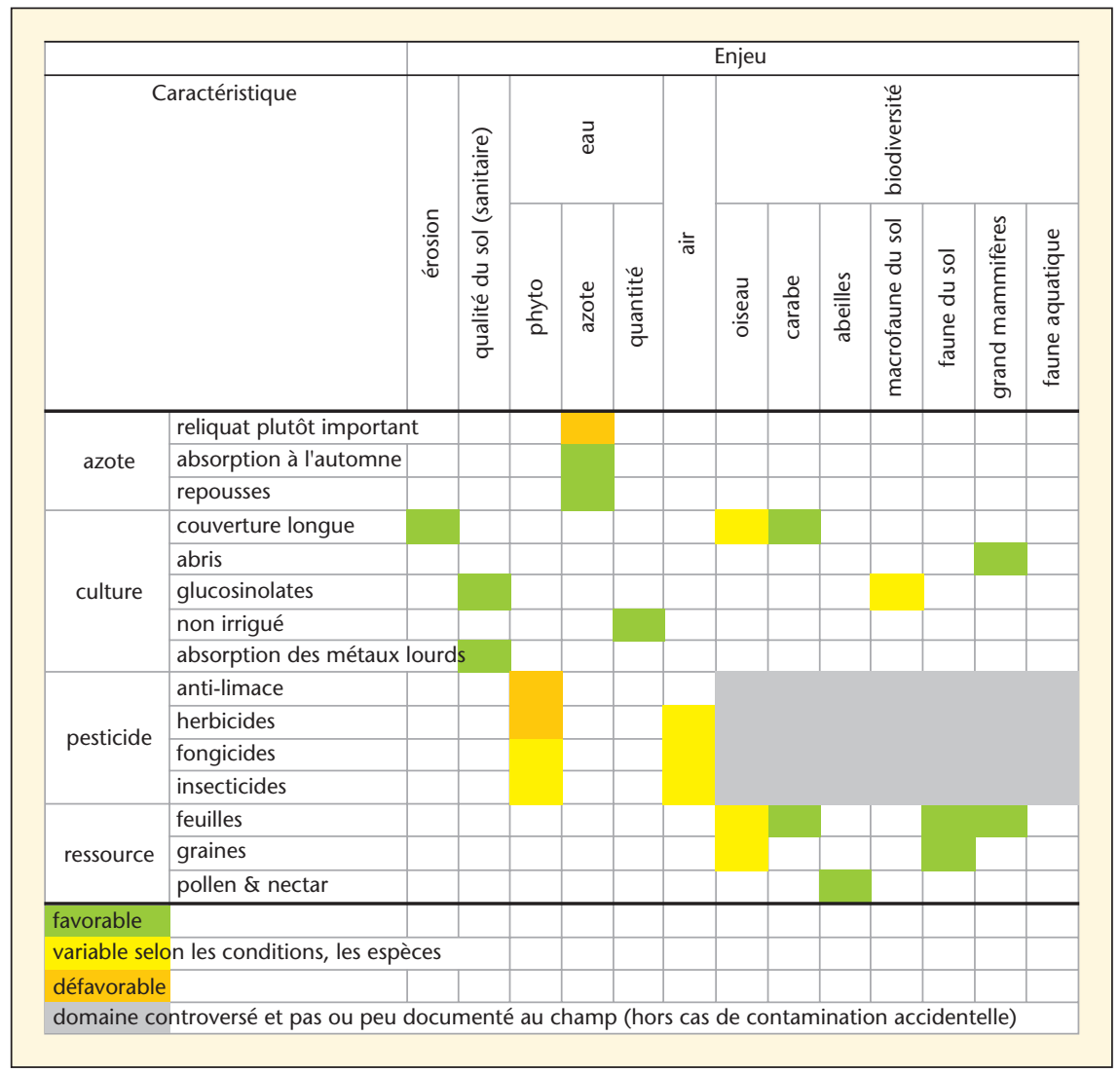

Figure 3. Influence des spécificités du colza sur quelques enjeux environnementaux.

Les impacts environnementaux peuvent aussi impliquer des flux entre parcelles (polluants, populations d'espèces animales, voire végétales). Ils sont mesurés dans ce cas à des échelles spatiales qui peuvent dépasser la parcelle: par exemple l'exutoire d'un bassin versant, ou le paysage pour des indices de biodiversité aviaire. De plus, ces impacts peuvent résulter d'effets cumulatifs. Le cas échéant, ils ne peuvent être interprétés sans informations d'ordre historique. Enfin, la mise en œuvre de solutions impose d'être attentif à la cohérence des systèmes de culture et des territoires pour éviter des effets contre-productifs. Le diagnostic et l'identification de solutions requièrent donc une approche systémique. La nécessité de travailler sur les systèmes de culture et les territoires remet en cause les approches centrées sur le produit, type ACV. Une utilisation abusive de ce type d'approche pourrait aboutir à des absurdités comme la promotion de la monoculture ou I'homogénéisation de I'utilisation du territoire autour de cultures jugées favorables. Autrement dit, la durabilité d'un territoire ne résulte pas de l'addition de la durabilité des éléments de ce territoire. La même règle vaut pour le système de culture et les cultures qui le composent.

Nonobstant ces difficultés, nous pouvons identifier des spécificités du colza qui peuvent être valorisées (figure 3): une couverture du sol longue, la fourniture de ressources trophiques variées, un potentiel assainissant du sol. La vraie question est de savoir comment. La meilleure réponse ne peut être que fonction d'un diagnostic prenant en compte les enjeux locaux, ainsi que les caractéristiques des systèmes de culture et des territoires.

\section{RÉFÉRENCES}

ATMO Poitou-Charentes. Mesure des phytosanitaires dans I'air en Poitou-Charentes Campagne 2009. Référence: PEST_INT_ 09_003; PEST_EXT_08_024. 
Bonnet JF, Lorne D. Eau et biocarburant. Impact sur l'eau du développement des biocarburants en France à I'horizon 2030. Les cahiers du CLIP $\mathrm{n}^{\circ}$ 19. Septembre 2009.

Burton NHK, Watts PN, Crick HQP, Edwards PJ. The effects of preharvesting operations on Reed Buntings Emberiza schoeniclus nesting in Oilseed Rape Brassica napus. Bird Study 1999; 46 : 369-372.

Burel F, Garnier E. Les effets de l'agriculture sur la biodiversité. In: Agriculture et Biodiversité. Valoriser les synergies. Expertise scientifique collective. Paris : Inra (France), 2008.

Doran JW, Parkin TB. Defining and assessing soil quality. In: Doran JW, Coleman DC, Bezdicek DF, Stewart BA (Eds.), Defining Soil Quality for a Sustainable Environment. SSSA special publication $\mathrm{N}^{\circ} 35$, Soil Sci Soc Amer Soc Agron, Madison, WI ; 3-21.

Faveret C. La place de I'allergie au pollen de colza parmi les pollinoses de printemps. Revue Française d'Allergologie et d'Immunologie Clinique ; 1976, 16 : 91-5.

Gallai N, Salles JM, Settele J, Vaissière BE. Economic valuation of the vulnerability of world agriculture confronted with pollinator decline. Ecological Economics 1999 ; 68 : 810 21.

Hole DG, Perkins AJ, Wilson JD, Alexander IH, Grice PV, Evans AD. Does organic farming benefit biodiversity? Biological Conservation $2005 ; 122$ : 113-30.

Holzshuh A, Dormann CF, Tscharntke T, Steffan-Dewenter I. Expansion of massflowering crops leads to transient pollinator dilution and wild plant pollination. Proceedings of the Royal Society B $2011 ; 278$ : 3444-51.
Kostioukevitch V. Du colza pour dépolluer Tchernobyl. Le Courrier International, 22 décembre 2010.

Ledoux E, Gomez E, Monget JM, et al. Agriculture and groundwater nitrate contamination in the Seine basin. The STICSMODCOU modelling chain. Science of The Total Environment 2007 ; 375 : 33-47.

Lerin J, Rivault E. Effet de la pollinisation entomophile sur le colza dans une expérience en cage. Agronomy 1982 ; 2 : 249-56.

Martin F, Lemanceau P. Rapport scientifique final ECOGER. Paris : Inra, 2008.

Mesquida J, Renard M. Pollinisation du colza $\mathrm{d}^{\prime}$ hiver mâle-fertile et mâle-stérile (Brassica napus L. var. oleifera metzger) par l'abeille domestique (Apis m. mellifica L.), effets sur la phénologie et le rendement. Apidologie $1981 ; 12$ : 365-62.

Mesquida J, Renard M, Pierre JS. Rapeseed (Brassica napus I.) productivity: the effect of honeybees (Apis mellifera I.) and different pollination conditions in cage and field tests. Apidologie $1988 ; 19:$ 51-72.

Moorcroft D, Wilson JD, Bradbury RB. Diet of nestling Linnets Carduelis cannabina on lowland farmland before and after agricultural intensification: Capsule Between the 1960s and 1990s, nestling diet has changed in ways consistent with the impact of agricultural intensification on the availability of seed sources. Bird Study 2006 ; 53 : 15662.

Pelosi C. Modélisation de la dynamique d'une population de vers de terre Lumbricus terrestris au champ. Thèse Agro Paris Tech, 2008.
Pierre J, Vaissière $B$, Vallée $P$, Renard $M$. Efficiency of airborne pollen released by honeybee foraging on pollination in oilseed rape: a wind insect-assisted pollination. Apidologie 2010 ; 41 : 109-15.

Pierre J, Renard M. Bilan de 30 ans de travaux de recherche effectués en France sur la pollinisation du colza. OCL 2010; 17 : 121-6.

Reau R, Bodet JM, Bordes JP, et al. Effets allélopathiques des Brassicacées via leurs actions sur les agents pathogènes telluriques et les mycorhizes : analyse bibliographique. OCL $2005 ; 12$ : 261-71.

Reau R, Wagner D. Des repousses de colza pour piéger les nitrates. Oléoscope $2000 ; 58$ : 6-8.

Sabbahi R, de Olivera D, Marceau J. Influence of honey bee (Hymenoptera: Apidae) density on the production of canola (Crucifera: Brassicacae). Journal of Economic Entomology $2005 ; 2$ : 367-72.

Sausse C. On the water footprint of biomass: a comment. Ecological Economics 2011 ; 71: 1-3.

Sausse C, Penillard E, Barbottin A, Jiguet F. Evaluation de l'impact de la culture du colza sur la biodiversité aviaire en France : enjeux, méthodes, premiers résultats. OCL $2011 ; 18$ : 145-52.

Sébillote M. Fertilité et systèmes de production. Paris : Inra, 1989.

Vidau C, et al. Exposure to sublethal doses of fipronil and thiacloprid highly increases mortality of honeybees previously infected by Nosema ceranae. PLOS ONE 2011; 6 : e21550. 